\section{Low serum vitamin D levels increase the risk of hip fracture in postmenopausal women}

Cauley et al. conducted a nested case-control study to investigate the relationship between serum 25-hydroxyvitamin D (25OHD) concentrations and risk of hip fracture in postmenopausal women. Previous studies had produced contradictory findings in relation to whether serum 25OHD levels affect fracture risk.

Participants were women aged $50-79$ years enrolled in the Women's Health Initiative Observational Study who did not have a history of hip fracture and who were not receiving boneactive agents or estrogens $(n=39,795)$. At study entry, participants provided information about their fracture risk factors and blood samples were obtained for the measurement of serum $250 H D$ levels. After a median follow-up of 7.1 years, 404 of the women had a hip fracture, of whom 400 were randomly selected as cases; controls were matched for age, ethnicity and date of blood sample collection.

Compared with women who had serum 25OHD concentrations in the highest quartile (70.7$121.5 \mathrm{nmol} / \mathrm{l})$, those with serum $25 \mathrm{OHD}$ concentrations in the lowest quartile $(9.2-47.5 \mathrm{nmol} / \mathrm{l})$ had a significantly increased risk of hip fracture. This association was independent of frailty, falls, physical functioning, renal function and sex-steroid hormone levels; however, higher levels of the bone resorption marker C-terminal telopeptide of type I collagen in cases than controls suggested a possible mechanism by which serum $250 H D$ level influenced fracture risk.

The authors suggest that measurement of serum 25OHD level in postmenopausal women might help to identify those at high risk of fracture.

Original article Cauley JA et al. (2008) Serum 25-hydroxyvitamin D concentrations and risk for hip fractures. Ann Intern Med 149: 242-250

\section{Gastric bypass improves metabolic syndrome components}

A healthy diet and exercise can improve the components of the metabolic syndrome in patients with insulin resistance, but the effect of weight loss alone was unknown. Now, researchers at the Mayo Clinic have found that weight loss by bariatric surgery considerably reduces the prevalence of the metabolic syndrome in a community-based cohort of obese individuals.

Batsis et al. retrospectively examined the outcomes for patients with a BMI $\geq 35 \mathrm{~kg} / \mathrm{m}^{2}$ who were evaluated for bariatric surgery between January 1990 and December 2003. Of the 337 patients, 180 underwent bariatric surgery (Roux-en-Y gastric bypass) and 157 did not; all patients received medical and dietetic care and advice about the importance of physical activity. Mean duration of follow-up was 3.5 years. Although metabolic syndrome prevalence decreased significantly in the nonsurgical group (from $85 \%$ to $75 \%$ ), a much more marked decrease occurred in the surgical group (from $87 \%$ to $29 \%$ ). Levels of obesity, hypertension, serum triglycerides and fasting glucose all fell significantly in the surgical group, but not in the nonsurgical group. Patients whose metabolic syndrome did not respond to surgery tended to lose less weight than responders, to be older, to have higher BMI, triglyceride and fasting glucose levels, and were more likely to have diabetes mellitus. Regression analysis suggested that \% excess weight loss was a major predictor of metabolic syndrome resolution.

The authors conclude that bariatric surgery is an effective means of treating the metabolic syndrome in eligible patients.

Original article Batsis JA et al. (2008) Effect of bariatric surgery on the metabolic syndrome: a populationbased, long-term controlled study. Mayo Clin Proc 83: 897-907

\section{Vitamin D deficiency is associated with myocardial dysfunction}

Vitamin D has been implicated in the control of myocardial calcium homeostasis and cardiac contractility. The worldwide prevalence of vitamin D deficiency is around $50 \%$ in the elderly and has been linked to arterial hypertension. Pilz and colleagues, therefore, investigated whether vitamin $\mathrm{D}$ deficiency might be associated with heart failure and sudden cardiac death (SCD).

Data were analyzed from the German Ludwigshafen Risk and Cardiovascular Health (LURIC) study, which enrolled patients referred for coronary angiography between July 1997 and 